\title{
Violence against Women: Methodological and Ethical Issues
}

\author{
Kaltrina Kelmendi ${ }^{1,2}$ \\ ${ }^{1}$ Department of Psychology, University of Prishtina, Prishtine, Kosovo \\ ${ }^{2}$ Faculty of Social Studies, Masaryk University, Brno, Czech Republic \\ Email: kaltrina.kelmendi@uni-pr.edu
}

Received April 18 ${ }^{\text {th }}, 2013$; revised May 22 ${ }^{\text {nd }}, 2013$; accepted June 20 ${ }^{\text {th }}, 2013$

\begin{abstract}
Copyright (C 2013 Kaltrina Kelmendi. This is an open access article distributed under the Creative Commons Attribution License, which permits unrestricted use, distribution, and reproduction in any medium, provided the original work is properly cited.
\end{abstract}

\begin{abstract}
Research on violence against women has improved and provides important information on patterns, prevalence, risk and consequences of this major threat to female well-being. Since the identification of violence against women as a problem worthy of study in 1970, evident progress has been made in understanding physical, psychological and sexual violence against women. However, while methodological improvements appear in later studies, the literature review shows many limitations and restrictions when conducting research on violence against women. The objective of this paper is to review the methodological issues that arise when studying violence against women. The paper focuses first on the history of research on violence against women, by elaborating on each perspective. Second, the paper identifies and describes methodological difficulties when researching violence against women such as methodology, operational definitions of violence, sampling frame and risk factors related to violence. The paper also elaborates on major ethical principles that should be considered and respected when researching violence against women. Finally, the paper recommends certain changes that should be made in order to improve future research on the subject.
\end{abstract}

Keywords: Intimate Partner Violence; Methodological Issues; Ethical Issues

\section{Introduction}

Violence against women, in its multiple forms, is increasingly recognized by individuals and states as a global problem and a serious violation of women's rights (Krug, Dahelberg, Mercy, Zwi, \& Lozano, 2002; Garcia-Moreno, Jansen, Ellsberg, Heise, \& Watts, 2006; UN Study, 2005; Ellsberg, 2006). Violence affects women in different ways; in particular on their mental and physical health. It leads to stress, depression, loss of self-esteem, reluctance to join the wider world, and more severe psychological and physical problems. The World Health Organization, which defines health as a state of complete physical, mental and social well-being, describes domestic violence by male partners as the most common health risk in the world for women (World Health Organization, 2002).

Intimate partner violence is the most common, widespread and predominant form of violence against women. In the WHO multi-country study conducted with over 24,000 women from 15 sites in 10 countries representing diverse cultural settings: Bangladesh, Brazil, Ethiopia, Japan, Namibia, Peru, Samoa, Serbia and Montenegro, Thailand, and the United Republic of Tanzania, it is estimated that the range of lifetime prevalence of physical violence by intimate partner was between 13\% (Japan city) and $61 \%$ (Peru province), with most sites falling between $23 \%$ and $49 \%$. Between $4 \%$ and $49 \%$ of ever-partnered women reported severe physical violence. The range of lifetime prevalence of sexual violence by intimate partner was between $6 \%$ (Japan city and Serbia and Montenegro) and 59\% (Ethiopia province), with most sites falling between $10 \%$ and $50 \%$. The range of lifetime prevalence of physical or sexual abuse, or both, by intimate partner was between 15\% (Japan city) and 71\% (Ethiopia province) (Garcia-Moreno, Jansen, Ellsberg, Heise, \& Watts, 2006: p. 1264). As could be noticed, researchers find considerable variation in the prevalence of partner violence from country to country and among studies within a country. Therefore, it is hard for researchers to compare international prevalence data on violence because different studies use different study populations, methods and definitions of violence.

This paper attempts to describe the history of research in the area of violence against women, through analyzing and examining the focus of research through the years. In addition, the paper aims to review methodological issues that arise when studying violence against women, focusing on: methods of data collection, operational definitions of violence, measures and risk factors. Furthermore, it tries to provide public opinion with ethical considerations and implications for conducting such a specific research. Finally, this review will focus on intimate partner violence, specifically on violence against women by male partners. For the purpose of this paper we define intimate partner violence as: "Any behavior within an intimate relationship that causes physical, psychological, or sexual harm to a partner in the relationship. Such behaviors include acts of physical aggression, psychological abuse, forced intercourse and other forms of sexual coercion, various controlling behaviors such as isolating a person from family and friends, monitoring her movements, and restricting access to information or assistance” (Heise \& Garcia-Moreno, 2002: p. 89). 


\section{Historical Background of Violence against Women}

Violence is a dynamic and complex phenomenon that transcends racial, ethnic, gender and age boundaries. Since family structure exists in all cultures, it is present in all cultures. Violence in families was not considered a problematic issue until the 1960s, due to cultural norms that refused to consider it a problem with consequences (Hotaling, Straus, \& Lincoln, 1990). Hence, intimate partner violence as a social or legal phenomenon was acknowledged only recently. The second wave of the feminist movement that emerged from the United States during the 1970s, and later from Western Europe, provided major contributions in this direction. The feminist movement originated from women who shared their life experiences of violent acts such as physical violence, rape and incest (Tjaden, 2005: p.1). As a result, women began to organize consciousness raising campaigns, write books, open shelters, and advocate reforms in laws regarding child abuse, domestic violence and sexual assault. Similarly, most of the scholars at that time focused on changing judicial systems to accomplish reform in the criminal codes defining sexual assault, domestic violence, and child abuse.

In the beginning of the 1980s violence against women began to be addressed as a public health issue. The public health perspective identified the prevalence and types of violence; it developed models that explained the violence and developed intervention programs for the treatment of victims (Carlson, Worden, Ryn, \& Bachman, 2000). However, most researchers doubted the usefulness of this approach due to the health sector's historic aim of diagnosis and treatment of individuals without raising social levels (Carlson, Worden, Ryn, \& Bachman, 2000). Despite the above mentioned doubts about the public health approach, the World Health Organization's report on violence against women was considered an important advancement among scholars in identifying types of interpersonal violence, such as: 1) physical violence, 2) sexual violence, 3) psychological violence, and 4) deprivation/neglect (Krug, Dahelberg, Mercy, Zwi, \& Lozano, 2002). Moreover, according to Klipatrick (2004), three important aspects were emphasized in the public health definition of violence: 1) intentional use of force or power (which refers to the tendency to harm the partner); 2) the intentional use of power or force (refers to the tendency to show power in a relationship, including: threats, intimidation and omission); and 3) these intentional acts are not required to produce injury, harm or deprivation in order to be defined as violence (p. 1214). Recently, violence against women has come to be recognized as a legitimate human rights issue and a significant threat to women's health and well-being (Ellsberg \& Heise, 2005). The identification of the link between violence against women and discrimination was a key to begin to consider the violence against women as human rights issue rather than criminal or public health issue (UN Study on Violence against Women, 2005). The acknowledgment of violence against women as a human rights issue was supported by evidence based research on the prevalence and incidence of violence and could be considered a major advancement. Multiple forms of violence against women indicated that violence against women is global and is based on the inequality between men and women. In this regard the human rights perspective sets out a number of measures, including those addressing traditional or religious practices, by which states might prevent and eliminate violence (UN Study on Violence against Women, 2005: p. 15).

\section{Methodological Issues on Researching Intimate Partner Violence}

Most of the studies done before the 1970s and at the start of the 1970's were conducted in clinical samples and used psychopathological models in their attempt to explain violence as a psychological disorder (Gelles, 1980). The first representative study, based upon the large sample of adult population, was conducted in the United States during 1975 and 1980. The aim of these studies was to obtain valid and reliable data on prevalence and causal analyses relying on statistical techniques. The results of this study (Gelles, 1980) and other studies (Straus, 1977, 1978; Steintmetz, 1977, 1978; Straus, Gelles \& Steinmetz, 1980) suggested that women are as violent as men toward their partners. Since then, researchers in the field of intimate partner violence have argued for gender symmetry or asymmetry of intimate partner violence. One side of the debate argued that men's use of violence against their partners differs from women's. Based on findings from national crime surveys (police, hospitals, shelters, courts) they considered that women are more likely than men to be victims of violence and to be injured during the intimate partner violence acts. According to these researchers, the violence is rooted in gender inequality and male dominance and there is no gender symmetry between the violent acts of men and those of women (Dobash R. E. \& Dobash R. P., 1977, 1978; Bograd, 1984; Dobash R. E., Dobash R. P., Wilson \& Daly, 1992).

The debate's influence on gender symmetry vs. asymmetry prevalence studies on intimate partner violence expanded greatly in the second half of the 1990s. The major contribution of research in the 90s was considered to be the distinctions of different types of violence (Johnson, 1995; Dobash R. E. \& Dobash R. P., 1992; Lloyd \& Emery, 2000; Riggs \& O’Leary, 1996; Tjadenn \& Thoennes, 1999) and the emphasis on "control”, (Johnson, 1995; Dobash \& Dobash, 1992; Pence \& Paymar, 1993) seen mainly in the feminist literature, which argues that intimate partner violence is a problem when men use violence to control their wives (as cited in Johnson \& Ferraro, 2000).

Recently the research in intimate partner violence, especially male violence against females, has increased. According to Garcia- Moreno \& Watts (2011), after the publication of findings from the WHO multi-country study in 2005, the research on intimate partner violence increased fourfold, from 80 to 300 in 2008. However many countries, as in the Middle East and West Africa, still lack reliable data (Garcia-Moreno \& Watts, 2011) and much of the existing information cannot be meaningfully compared.

A population-based survey is considered the most reliable method for obtaining information on violence against women in a general population. Since it uses randomly selected samples, its results are representative of the larger population and it accurately provides estimates of the prevalence of violence against women. The population based surveys that most countries use are of two types: dedicated surveys designed to gather detailed information on different forms of violence against women, and larger scale surveys (generic surveys) designed to gather data on broader issues such as poverty, crime or reproduction, but also have questions or a module of questions on 
violence against women. According to Walby and Myhill (2001), many national representative surveys on violence against women and domestic violence have been conducted all over the world during the 1990s, particularly in Europe and North America, such as The National Violence against Women survey conducted in Australia, France, Germany, Sweden and the United States of America. Based on the findings it seems that generic or dedicated surveys have a strength and a weakness (Walby \& Myhill, 2001). The strength of the generic survey is that it covers a lot of people for a certain period of time, but there is a dilemma regarding the use of shorthand and screener questions, since it might hinder the victim's disclosure if she has not been previously identified as a victim. Dedicated surveys were shown to be more effective in collecting data on violence against women, since their methodology had been previously adapted to the issue, but their disadvantage is related to lack of funding and resources.

Furthermore, the interviewing process in surveys is considered an important step toward enhancing the disclosure of violence by sharing the story of abuse and recovery. In this regard, Walby and Myhill (2001) identified three important aspects: privacy, interviewing skills and gender of the interviewer. It has been proven that when the interview is done with nobody present; when the interviewer is female and specially trained, the disclosure rate is higher. For instance, in the British Crime Survey, when the woman's partner was involved in completing the questionnaire the rate of reporting lifetime violence dropped to less than half the rate reported when no one else was present, from $23 \%$ to $10 \%$ (Walby, 2000). Similarly, Sorenson et al. (1978) found that those interviewed about sexual assault were 1.27 times more likely to reveal a sexual assault if they were interviewed by a woman than by a man.

Standard methodology for the implementation of surveys on violence against women within the framework of official statistics has not yet been developed at the international or supranational level (UN Study on Violence against Women, 2005). For example, many of the prevalence estimates for intimate partner violence are not comparable because of methodological differences in how violence has been defined and measured. In this regard Tjaden (2005) mentions three factors related to lack of accurate data in these surveys: narrow focus, differing time frame and inadequate measurement. It should be emphasized that there is an ongoing effort of international organizations to support internationally comparative surveys. In this regard, two important studies should be mentioned: the International Violence against Women Surveys (IVAWS) coordinated by the European Institute for Crime Prevention and Control and the WHO Multi-Country Study on Domestic Violence and Women's Health. The IVWAS has been conducted in ten countries (Australia, Denmark, Canada, England, Wales, Finland, Italy, Netherlands, Sweden and Switzerland). It is a comparative study specifically designed to target's men's violence against women, including physical and sexual violence (Johnson, Ollus, \& Nevala, 2008). The WHO Multi-Country Study has been conducted in 15 cities and 11 countries (Bangladesh, Brazil, Ethiopia, Japan, Namibia, Peru, Samoa, Serbia and Montenegro, Thailand, and the United Republic of Tanzania) and its aim was to collect data on intimate partner violence, sexual assault, child abuse and the consequences of violence (Garcia- Moreno, Jansen, Ellsberg, Heise, \& Watts, 2006).

In addition to population studies, service based studies are considered another source of information on violence against women. Service based studies include data about violence against women gathered through public and private agencies, supporting women who have experienced violence, including: health centers, police stations, shelters, legal and advocacy organizations and lawyers' associations. Although these data could not be used for measuring prevalence of the phenomena, they contribute to understanding risk factors that influence violence and help in designing adequate policies to protect women on the supranational level (UN Study on Violence against Women, 2005).

Qualitative studies are considered another important and necessary method of data collection on violence against women. Mostly, the qualitative studies are based on in-depth interviews, focus groups or participatory research (Michau, 2002; Kilonza et al., 2003; Sagat, 2000; Zimmerman, 1995 cited in Ellsberg \& Heise, 2005: pp. 74-81). Taking into account that qualitative research is considered useful for describing complex phenomena, its major strengths in understanding intimate partner violence are: collection of data on women's experiences of violence in their words; their thoughts on the causes of violence; rich details describing the cultural and local context in which violence occurs; understanding of contextual and setting factors as they relate to the intimate partner violence; a naturalistic setting of data collection and the women's interpretation of intimate partner violence. Findings from qualitative research are mainly presented through narratives, case studies, descriptions and quotes. In addition, qualitative studies are of important relevance in designing preventive campaigns, evaluating and monitoring interventions and engaging the community in fighting violence against women (UN Study on Violence against Women, 2005).

\section{Sampling Frame}

There are great variations from year to year in the population studies used for researching violence against women. In the beginning of the 70's most research on violence against women was based on non-representative clinical samples. Only in the late 70's the first study was done with representative samples (Gelles, 1980). According to the findings of this study (Straus, 1977, 1978) and studies conducted by Strauss, Gelles, Steinmetz Gelles (1980) and Steinmentz $(1977,1978)$ the gender neutral view of intimate partner violence became popular, which suggested that females are equally violent as males in relationships. Similarly, feminist scholars at that time conducted studies on the specific factors related to male violence against women. Feminist theoerists suggested that there is no gender symmetry in perpetration of violence and only men are socialized to believe that perpetrating violent behavior is acceptable in romantic relationships (Johnson, 1995). Furthermore, according to the feminist approach, male violence against females is situated within the history of family and larger context of gender inequalities that gives a man the right to dominate the home and subordinate his partner (Yllo \& Bograd, 1988 cited in Johnson, 1995: p. 283). The feminist approach relied on research designs that collected data with those agencies that came in contact with hospitals, shelters and other relevant stakeholders.

However, even though the methodology used in populationbased surveys is considered the most reliable for collecting accurate data on violence against women, these studies have been subject to many critics, especially feminist scholars 
(Johnson, 1995; Johnson \& Ferraro, 2000; Johnson \& Leone, 2005; Johnson, 2006). Although the survey aimed to gather information from target samples, according to feminist scholars non-respondents' responses may have differed drastically from the respondents' responses (Johnson, 1995). Additionally, the accuracy of the gathered data depends on the response rate. A man who assaults his wife probably will not participate in the survey, whereas the battered wife will be scared to answer the questions. Conversely, according to Ruiz-Perez et al. (2007), the weakness of clinical based samples (hospitals, shelters) lies in the data gathered from reported cases that experienced the most severe type of violence, and the effect of trauma will obstruct their responses as well. Accurate data on violence are lacking, since the less severe cases of violence are excluded.

Furthermore, most of the national surveys tend to exclude certain populations from the studies such as temporary residents of shelters and hostels. These particular population groups are more likely to be subject to violence than those who are included in the sampling frame. Additionally, these women are more likely to experience violence in its aftermath than the general population. Therefore, the exclusion of these categories under-represents the accurate rate of violence in national samples (Walby \& Myhill, 2001).

\section{Operational Definition of Violence}

The most difficult and controversial issue in studying violence against women is related to its operational definition. This happens because most common terminology comes from diverse theoretical perspectives and has different meanings in different regions (Walby \& Myhill, 2001). However, despite the acknowledgment of scholars on the need for uniformity in definitions and measurements of violence against women, there is no formal mechanism for arriving at that desired uniformity (Saltzman, 2004).

In the beginning of 1970, due to increased interest in the issue of violence and its varieties, many studies have been conducted and the main aim of these studies was to refute the general conception that violence happens due to psychological disorders, and to provide opinion with valid and reliable data. Therefore, the researchers tried to improve the methodological techniques on data collection and tried to expand knowledge on the explanation of violence. However, despite their struggle to provide reliable and valid data on domestic violence, research (Kempe et al., 1962; Straus et al., 1980; Giovannoni \& Becerra, 1979 ) in the 70's was facing many problems regarding the conceptualization of violence and its measurement (as cited in Gelles, 1980).

Additionally, according to Klipatrick (2004), each perspective given in definition of violence against women historically contains some problematic issues, especially regarding the narrowness of focus. For instance, the criminal justice perspective is considered a narrow definition since it includes only violent acts that are defined legally as crime, ignoring other violent acts such as psychological or emotional abuse and deprivation. Because public health perspective includes non-violent acts such as emotional/psychological abuse, deprivation and neglect, it is considered broader compared to criminal justice perspectives, but the tendency to focus on sexual abuse rather than other types of violence is considered narrow and problematic. Finally, the human rights perspective is considered broader since it includes all types of violent crimes against women: psychological abuse, harmful traditional practices such as genital cut- ting, forced marriages and state tolerated discrimination against women.

Feminist scholars suggest that even broader definitions of violence against women should be used, since narrow definition lowers incidence and prevalence of phenomena (DeKeseredy, 2000 as cited in Kilpatrick, 2004: p. 11). According to feminist scholars, we have to be very cautious when measuring intimate partner violence since we have to differentiate between two types of intimate partner violence: common violence that refers to conflicts in the relationship and patriarchal terrorism that uses violence in order to show power and control over the female (Johnson \& Ferraro, 2000, Johnson, 2011 ). Failure to make such distinctions could lead to incorrect estimates regarding the frequency of common couple violence against men and the conclusion that there is a widespread "battered husband" syndrome (Johnson, 1995: p. 292). Recent papers (Johnson \& Ferraro, 2000; Johnson \& Leone, 2005; Johnson, 2006) make further distinctions between intimate partner violence such as Intimate Terrorism, Violent Resistance, Mutual Violence Control and Situational Couple Violence. Intimate Terrorism refers to a relationship where only one of the partners is violent and controlling, and the other partner is either nonviolent or violent but not controlling. Violent Resistance refers to cases when one of the partners is violent but not controlling, while the other partner is violent and controlling. Situational Couple Violence refers to non-controlling violence that occurs in a relationship between partners. Mutual Violence Control refers to controlling violence between partners whereas both partners are violent and controlling. Furthermore, findings from the study conducted by Graham-Kevan \& Archer (2003) on a mixed population and a shelter sample, using a CTS style questionnaire, support earlier findings from the study conducted by Johnson \& Ferraro (2000). Based on these findings (87\%) of intimate partner violence was perpetrated by males, while common couple violence was almost symmetrical, perpetrated by (45\%) males and (55\%) females. As was expected, the shelter population experienced $70 \%$ of all intimate partner violence, while $94 \%$ of common couple violence was found in the community sample. Finally, over the past decade, there is a growing body of research that demonstrates the existence of different types of intimate partner violence such as (Graham-Kevan \& Archer, 2003; Johnson, 1995; Johnson \& Leone, 2005; Johnson \& Ferraro, 2000; Johnson, 2006, Kelly \& Johnson, 2008).

Similarly, Ruiz- Perez et al. (2007) and Walby (2004), referring to the UN Declaration on Elimination of Violence Against Women, consider that, although the broader definition of violence is complex and includes most of the women's violent experiences, the terms used within the definition tend to lose their power and meaning.

\section{Measuring Violence against Women}

There are many instruments used to measure violence against women such as: Conflict Tactic Scale; Psychological Maltreatment of Women Index; and Measure of Wife Abuse and Index of Spouse Abuse (Hegarty, Sheehan, \& Schonfeld, 1999). The Conflict Tactic Scale developed by Strauss and Gelles (1979) is considered the most widely used measure for assessing intimate partner violence and is the only standardized instrument (Dietz \& Jasinski, 2007). The original CTS scale measured acts used in conflicts, verbal and physical aggression and it focused on the severity and frequency of such acts. In 
addition, this scale is used to measure husband to wife violence, wife to husband violence and parent to child violence. The revised version, which is called CTS2, includes also some questions on sexual violence (Ellsberg \& Heise, 2005). Besides its wide usage, the CTS scale was the object of many criticisms. First it focuses mainly on physical abuse, with some items on verbal aggression, while it ignores other forms of violence, especially psychological violence (Hegarty, Sheehan, \& Schonfeld, 1999). In addition, controversial findings obtained from larger scale surveys concluded that women are as violent as men and suggested so-called gender symmetry. Feminist scholars stated that these findings are opposite to the results gained from the studies done in shelters and social services, since the scale is not able to distinguish among types of interpersonal violence (Johnson, 1995). Furthermore, most of the research suggests that female violence is rather related to selfdefense than a tendency to injure somebody (Ellsberg \& Heise, 2005). CTS has also been criticized for not taking into consideration the context, meaning and motive of the violent acts (Schwartz, 2000) and their impact, since the same acts may cause different injuries and have different meanings for men as for women (Walby, 2001).

Nevertheless, other instruments were also predisposed toward criticism. For instance, Psychological Maltreatment of Women mainly focuses on emotional abuse and ignores other forms of violence. The Index of Spouse Abuse and Measure of Wife Abuse is a 30-item self-report scale that focuses on broader aspects of partner violence, respectively in physical and non-physical violence. However, both of them were validated in small samples (Hegarty, Sheehan, \& Schonfeld, 1999). Recently, a WHO questionnaire has been designed primarily for measuring violence against women based on the previous research experiences. This questionnaire gathers data on physical, sexual and emotional forms of violence. In addition, it provides us with the frequency of violence, duration of violence, health consequences and response to abuse (Ellsberg \& Heise, 2005).

Finally another problematic aspect, of instruments used to measure violence against women, is related to different time frames in which violence has been measured (Tjaden, 2005; Walby \& Myhill, 2001). In addition, according to Walby (2001) the prevalence and incidence should be defined separately. Some of the instruments ask about the violence experienced during the person's lifetime while others ask about just the last year. As a result, comparisons between the findings would be impossible. Furthermore, the recall bias in surveys asking about violence experienced during one's lifetime would lead to an underestimation of violence, while the response rate for the last year will give more accurate estimates of violence.

\section{Risk Factors Related to Violence against Women}

There is no single, definitive cause of family violence, and many people-regardless of gender, age, race, ethnicity, education, cultural identity, socioeconomic status, occupation, religion, sexual orientation, physical and mental abilities or personality - may be vulnerable to abuse at any stage of their lives. There are numerous factors associated with violence and findings from the study WHO multi country study on women's health and domestic violence found that many similarities across sites in terms of risk factors for intimate partner violence (Abramsky, Watts, Garcia-Moreno, Devries, Kiss, Ellsberg,
Jansen \& Heise, 2011; Heise, 2012). However, an ecological model could be considered a most useful model to explain and describe diverse factors related to each other, and could classify factors as follows: socio-cultural risk factors, social structural risk factors, family factors and individual factors for victims and perpetrators. Many studies were conducted to identify individual risk factors related to violence; however most of the research on risk factors for violence is focused on male perpetrators (Tolan, Gorman-Smith, \& Henry, 2006). According to Kantor and Jasinski (1998) male perpetrators are characterized by: low self-esteem and low impulse control; anxiety disorders, depression, antisocial personality disorder and substance abuse, poor social skills and insecurity (as cited in (Carlson, Worden, Ryn, \& Bachman, 2000; Tolan, Gorman-Smith, \& Henry, 2006). In addition sexual abuse as a child has been identified as a risk factor in males for sexual offending (Crowell \& Burgess, 1996). Furthermore, the experience of violence in the family and corporal punishment as a child are considered as risk factors for intimate partner violence as an adult (Riggs \& O’Leary, 1996; Abramsky et al., 2011). Age is identified as another individual risk factor for victims and perpetrators; the risk is higher for younger partners. Hence, when analyzing the risk factors for victims there is always some doubt if these factors are consequences of victimization or risk factors as such. This ambiguity is especially related to social isolation and substance abuse (Crowell \& Burgess, 1996).

Family risk factors are related to relationship factors and dynamics within the family or couple that lead to violence. There is little research done in this regard. However some of the identified factors are: poor problem-solving and communication skills, relationship status, and economic dependency of females (Carlson, Worden, Ryn, \& Bachman, 2000). Stress is considered another risk factor for violence against women, especially when the family experiences poverty, immigration, discrimination or medical problems (Levy, 2008). Moreover, based on the findings of a UN Study (2005) the following factors were identified: male control of wealth and decision-making authority within the family; a history of marital conflict; and significant interpersonal disparities in economic, educational or employment status.

Social structural risk factors are related to economic status and community factors. Most research concludes that poverty; low-income rates and unemployment of males could be considered risk factors. Community risk factors mainly related to services offered for victims of violence, the stigma accompanying these services, and the phenomenon of violence in general (Carlson, Worden, Ryn, \& Bachman, 2000). Furthermore, findings from the UN Study (2005) identify similar and different factors related to the community aspect: women's isolation and lack of social support; community attitudes that tolerate and legitimize male violence; and high levels of social and economic disempowerment.

Socio-cultural risk factors, according to the findings of most studies, relate to cultural norms and traditions that promote the so called culture of acceptance of violence, which could be considered another risk factor for victimization. Some of the studies find that patriarchal families, cultures accepting of violence and gender stereotypes are considered major risk factors (Crowell \& Burgess, 1996). The UN Study (2005) identifies gender roles, male dominance and tolerance of violence for conflict resolution as major risk factors related to socio-cultural level. 


\section{Ethics in Researching Violence against Women}

Research on violence against women is a very difficult and challenging process due to the sensitive nature of the phenomenon. Despite the sensitivity, reliable information on violence against women is a crucial element for prevention, treatment and elimination of different forms of violence. In this regard WHO, when conducting research on gender based violence in 1999, published guidelines for safety and ethics, addressing the safety of both respondents and interviewers; ensuring the privacy and confidentiality of the interview; providing special training to interviewers on gender equality issues and violence against women; providing information and referrals for respondents in situations of risk; and providing emotional and technical support for interviewers (UN Study on Violence against Women, 2005: p. 60).

According to Ellsberg and Heise (2002), some major ethical principles should be taken into account when conducting research on gender based violence: non-malfeasance, beneficence, respect for individuals and justice (balancing risks and benefits).

The major ethical concern in studies on violence against women is related to the principle of non-malfeasance or minimizing the harm. This principle is related to the fact that respondents who participate in the study are more likely than others to suffer physical harm if their partners find out the aim of the study. WHO suggested some alternatives for minimizing harm to respondents: interviewing only one woman per household; not informing the wider community that the study includes questions on violence; not conducting research with men in the same clusters while women are interviewed; conducting the interview in complete privacy; using dummy questionnaires if somebody enters the room; using candy and games to distract children; providing information on services available to protect victims of violence (Ellsberg \& Heise, 2005: p. 39). Additionally, taking into consideration that interviews about violence may provoke intense emotions and painful feelings, the interviewers should be trained to respond in such situations and end the interview by emphasizing the woman's strengths.

The principle of beneficence for research on violence relates to the maximizing of benefits for respondents that participate in the study. Telling the story of violence is considered a transforming experience for the women, since they did not have an opportunity to talk about it before (Ellsberg \& Heise, 2005: p. 43). Another aspect of beneficence relates to methodological considerations that maximize disclosure, including: specific wording and templates for questions; interviewers' sex; attitude; skills; empathy and trust. Finally, the major consideration of the beneficence principle is associated with findings that lead to social change. According to WHO guidelines, researchers and donors have an ethical obligation to help ensure that their findings are properly interpreted and used to advance policy and intervention development (Ellsberg \& Heise, 2002: p. 39). Additionally, the principle of respect for individuals is considered an important ethical principle that should be taken into account when conducting research on violence against women. Respect involves two important considerations: respect for autonomy and protection of vulnerable people. These considerations relate to individual informed consent procedures that ensure respondents understand the purpose of the research and that their participation is voluntary (Ellsberg \& Heise, 2005: p. 36). Besides, there is ongoing debate among researchers regarding the ade- quate form of informed consent, since some of the researchers believe it is important to inform participants regarding the questions on violence, whereas other researchers think this will hinder the disclosure and increase the respondents' anxiety. Finally, the principle of justice in research is related to balancing the risks and benefits to women who participate in the study. In this regard, the risk of women participating in the study is fairly large, but the risk of silence, ignorance and inaction toward the violence is large as well. Therefore the researchers are guided continuously to balance these two realities (Ellsberg \& Heise, 2005).

\section{Improving Future Studies}

Research on violence against women has provided important information regarding patterns, prevalence, risk and consequences of this threat to female well being. Since the identification of violence against women as a problem worthy of study in 1970 , progress has been made in understanding physical, psychological and sexual violence against women. Additionally, the research on violence against women in the past two decades has improved and is considered a crucial element for designing prevention and treatment programs for violence against women. Although methodological improvements have been made in later studies, a review of the literature shows many limitations and restrictions that should be taken into account when researching violence against women.

Taking into consideration the phenomenon's complexity, when conducting research on violence against women a mixed methodology should be used, combining qualitative and quantitative methods. In this regard, quantitative methods will produce information that could be summarized in numbers and percentages of women who experienced violence, while qualitative methods will enable detailed and in-depth information to be gathered from the experiences of violence. Consequently the combination of both methods would provide a truthful picture. Recently researchers on violence against women encourage the use of more than one method when analyzing the same issue. Furthermore, considering that the research's aim relies on understanding the phenomenon of violence against women and designing effective preventive and awareness-raising programs, I think that intimate partner violence should be studied more carefully and as a product of the social processes, contexts and interactions in which the phenomenon exists. Intimate partner violence is a subjective and personal issue and the knowledge that we gain through mono-method studies, particularly quantitative studies, is rather vague and superficial. Therefore, I would strongly recommend the analysis of intimate partner violence through the assessment of social contexts, individual differences and multiple realities based on social, political, cultural, ethnic, gender and disability values. I think that through such analysis a deeper and richer understanding will be reached. Integrating qualitative methods in the study of intimate partner violence will enhance understanding of the problems and research findings, interpret inconsistent results and advocate for social change.

\section{REFERENCES}

Abramsky, T., Watts, Ch. H., Garcia-Moreno, C., Devries, K., Kiss, L., Ellsberg, M., Jansen, H., \& Heise, L. (2011). What factors are associate with recent intimate partner violence? Findings from the WHO 


\section{K. KELMENDI}

multi-country study on women's health and domestic violence. BMC Public Health, 11, 109. doi:10.1186/1471-2458-11-109

Bogard, M. (1984). Family systems approaches to wife battering: A feminist critique. Americal Journal of Orthopsychiatry, 54, 559-568.

Carlson, B. E., Worden, A. P., Ryn, M., \& Bachman, R. (2000). Violence against women: Synthesis of research for practitioners. Final Report submitted to the National Justice, grant number 98-WTVX-K011. Washington, DC: US Department of Justice.

Crowell, N. A., \& Burgess, A. W. (1996). Understanding violence against women. Washington, DC: National Academies Press. doi:10.1037/10204-000

Dobash, R. E., \& Dobash, R. P. (1977, 1978). Wives: The “appropriate" victims of martial violence. Victimology: An International Journal, 2, 426-442.

Dobash, R. E., Dobash, R. P., Wilson, M., \& Daly, M. (1992). The myth of sexual symmetry in marital violence. Social Problems, 39, 71-91. doi:10.2307/3096914

Dietz, T. L., \& Jasinski, J. L. (2007). The evect of item order on partner violence reporting: An examination of four versions of the revised Conflict Tactics Scales. Social Science Research, 36, 353-373. doi:10.1016/i.ssresearch.2006.04.006

Ellsberg, M., \& Heise, L. (2002). Bearing witness: Ethics in domestic violence research. Violence against Women, 359, 1599-1604.

Ellsberg, M., \& Heise, L. (2005). Researching violence against women: A practical guide for researchers and activists. Washington, DC: World Health Organization.

Ellsberg, M. (2006). Violence against women: A global public health crisis. Scandinavian Journal of Public Health, 34, 1403-4948. doi:10.1080/14034940500494941

Garcia-Moreno, C., Jansen, H. A., Ellsberg, M., Heise, L., \& Watts, C. H. (2006). Prevalence of intimate partner violence: Findings from the WHO multi country study on women's health and domestic violence. Geneva: World Health Organization, Department of Gender, Women and Health.

Garcia-Moreno, C., \& Watts, Ch. (2011). Violence against women: An urgent public health priority. http://www.who.int/bulletin/volumes/8 /1/10-085217.pdf

Gelles, R. J. (1980). Violence in the family: A review of research in the seventies. Journal of Marriage and Family, 42, 873-885. doi: $10.2307 / 351830$

Heise, L., Ellsberg, M., \& Gottemoeller, M. (1999). Ending violence against women. Population Information Programme, 27.

Heise, L. (2012). Determinants of partner violence in low and middle income countries: Exploring variation in individual and population -Level risk. PhD Thesis, London: London School of Hygiene Tropical Medicine.

Hegarty, K., Bush, R., \& Sheehan, M. (2005). The composite abuse scale: Further development and assessment of reliability and validity of a multidimensional partner abuse measure in clinical settings. Violence and Victims, 20, 529-547.

Hotaling , G. T., Straus, M. A., \& Lincoln, J. (1990). Intrafamily violence, and crime and violence outside the family. Family Violence, 11.

Johnson, M. (1995). Patriarchal terrorism and common couple violence: Two forms of violence against women. Journal of Marriage and Family, 57, 283-294. doi:10.2307/353683

Johnson, M. (2006). Conflict and control: Gender symmetry and asymmetry in domestic violence. Violence against Women, 12, 1003. doi:10.1177/1077801206293328

Johnson, M., \& Ferraro, K. J. (2000). Research on domestic violence in the 1990s: Making distinctions. Journal of Marriage and Family, 62, 948-963. doi:10.1111/j.1741-3737.2000.00948.x

Johnson, M., \& Leone, J. M. (2005). The differential effects of intimate terrorism and situational couple violence: Findings from the national violence against women survey. Journal of Family Issues, 26, 322. doi:10.1177/0192513X04270345

Johnson, H., Ollsun, N., \& Nevala, S. (2008). Violence against women:
An international perspective. Berlin: Springer.

Johnson, M. P. (2011). Gender and types of intimate partner violence: A response to an anti-feminist literature review. Aggression and Violent Behaviour, 16, 289-296. doi:10.1016/j.avb.2011.04.006

Kilpatrick, D. G. (2004). What is violence against women: Defining and measuring the problem. Journal of Interpersonal Violence, 19, 1209. doi: $10.1177 / 0886260504269679$

Kelly, J. B., \& Johnson, M. P. (2008). Differentiation among types of intimate partner violence: Research update and implications for interventions. Family Court Review, 46, 476-499. doi:10.1111/j.1744-1617.2008.00215.x

Krug, E. G., Dahelberg, L. L., Mercy, J. A., Zwi, A., \& Lozano, R. (2002). World report on violence and health. Geneva: World Health Organization.

Riggs, D. S., \& O’Leary, K. D. (1996). Aggression between heterosexual dating partners: An examination of a courtship model of courtship aggression. Journal of Interpersonal Violence, 11, 519-540. doi:10.1177/088626096011004005

Ruiz-Perez, I., Plazaola-Castano, J., \& Vives-Cases, C. (2007). Methodological issues in the study of violence against women. Journal of Epidemiol Community Health, 61, ii26-ii31. doi:10.1136/jech.2007.059907

Saltzman, L. E. (2004). Issues related to defining and measuring violence against women response to Kilpatrick. Journal of Interpersonal Violence, 19, 1235. doi:10.1177/0886260504269680

Schwartz, M. D. (2000). Methodological issues in the use of survey data for measuring and characterizing violence against women. Violence against Women, 6, 815-838. doi:10.1177/10778010022182164

Steinmetz, S. K. (1977, 1978). The battered husband syndrome. Victimology, 2, 499-509.

Straus, M. A. (1977, 1978). Wife beating: How common and why? Victimology, 2, 443-458.

Straus, M. A., Gelles, R. J., \& Steinmetz, S. K. (1980). Behind the closed doors: Violence in the American family. Garden City, NY: Doubleday.

Sorenson, S. B., Stein, J. A., Siegel, J. M., Golding, J. M., \& Burnham, M. A. (1978). The prevalence of adult sexual assault: The Los Angelos epidemologic center catchment area project. America Journal of Epidemiology, 125, 1154-1164.

Tjaden, P. (2005). Violence against women: A statistical overview, challenges and gaps in data collection and methodology and approaches for overcoming them. Geneva: World Health Organization, UN Division for the Advancement of Women.

Tjaden, P., \& Thoennes, N. (1998). Prevalence, incidence, and consequences of violence against women: Findings from the national violence against women survey. Research in Brief. Washington, DC: US Department of Justice, National Institute of Justice, and US Department of Health and Human Services, Centers for Disease Control and Prevention.

Tolan, P., Gorman-Smith, D., \& Henry, D. (2006). Family violence. Annual Review of Psychology, 57, 557-583. doi:10.1146/annurev.psych.57.102904.190110

UN Study on Violence against Women (2005). In-depth study on all forms of violence against women. 61st Session of the General Assembly Item 60(a) on Advancement of women. http://www.un.org/womenwatch/daw/vaw/SGstudyvaw.htm

United Nations General Assembly (1993). UN declaration on the elimination of violence against women. New York: United Nations General Assembly.

Walby, S. (2001). Improving the statistics on violence against women. Statistical Journal of the United Nations, 22, 193-216.

Walby, S., \& Myhill, A. (2001). New survey methodologies in researching violence against women. British Journal of Criminology, 41, 502-522. doi:10.1093/bjc/41.3.502

World Health Organization (2002). Reducing risks promoting health life. Geneva: World Health Organization. 